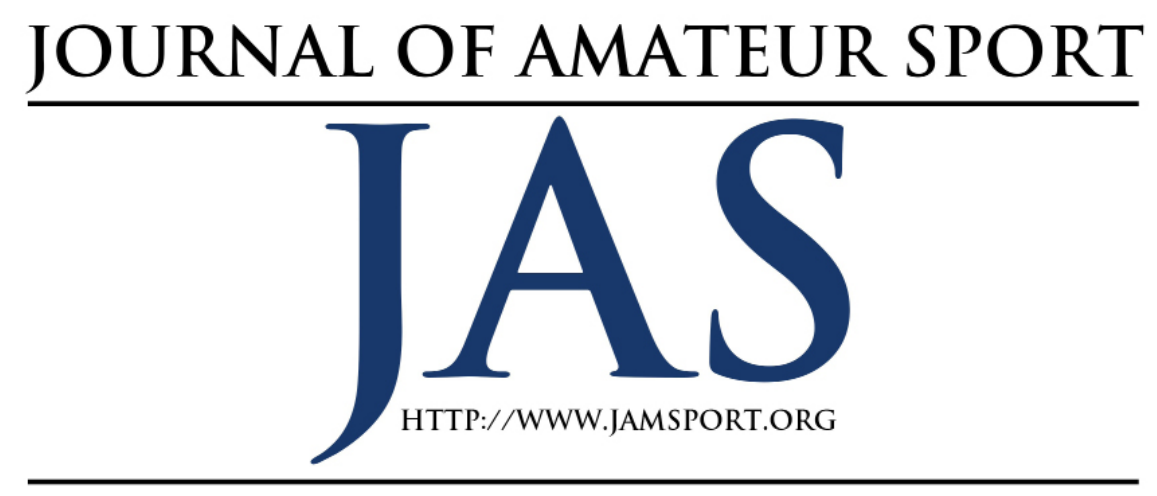

\title{
Parent Perspectives on Youth Sport Concussion Management
}

\author{
Corinne M. Daprano ${ }^{1} \quad$ Elana R. Bernstein ${ }^{1}$ \\ Susan C. Davies ${ }^{1}$
}

${ }^{1}$ University of Dayton

Concussions are a key health concern at all levels of sport. While much of the discussion focuses on American football and the National Football League (NFL), there is growing awareness in the sport community that the risks of concussions extend to head injuries in all sports. Much remains unknown about youth concussions, particularly in terms of how parents feel about concussions and youth sport safety regulations. Therefore, the purpose of this study was to examine parent's perceptions and attitudes toward: 1) youth sport concussions; 2) the efficacy of return-to-learn and return-to-play educational resources; and, 3) a parent's role managing their child's return to school and play after a concussion. Findings indicated that despite increased awareness and education there remain gaps in parental knowledge of concussions and the proper management of concussions. These findings lead to a set of recommendations regarding parental concussion prevention and management education.

\section{Introduction}

$\mathrm{I}$ $\mathrm{n}$ a report compiled by the Institute of Medicine (IOM) and National Research Council (NRC) of the National Academies, researchers noted that there is still much that is unknown about the diagnosis, management, and prevention of concussions (IOM, 2014). There are several reasons children may experience a concussion, including through sport participation. According to results of a national sample of US high school sports, approximately $70 \%$ of concussions among high school 
athletes were caused by player-to-player contact and another $17 \%$ by player-toplaying surface contact (Marar, McIlvain, Fields, \& Comstock, 2012).

The highest rates of concussions for male high school athletes occur in football, ice hockey, lacrosse, wrestling, and soccer. For female high school athletes, the highest concussion rates occur in soccer, lacrosse, basketball, and ice hockey (IOM, 2014). Many parents view injuries, such as being hit by a baseball or a fall during a sport activity, as a typical childhood incident and may be reluctant to consult with a medical professional after this type of an incident (Lin et al., 2015, Macdonald \& Hauber, 2016). Parents may also be reluctant to consult with a medical professional because they lack knowledge of concussion symptoms and treatment.

In general, several studies have found that parents have moderate knowledge of concussion symptoms. The most commonly identified symptoms were physical symptoms (Coghlin, Myles, \& Howitt, 2009; Gourley, Valovich McLeod, \& Bay, 2010). Yet, parents often lack knowledge regarding return-to-learn and return-to-play procedures following a concussion. According to Sullivan et al. (2009), parents understand that concussions are a serious health risk and the dangers associated with them, but they lack knowledge of specific return-toplay protocols. Turner, Lucas, Margolis \& Corwell (2017) found that while parents have general knowledge about concussions, they typically are not familiar with return- to-play rules, procedures and educational resources such as the Centers for Disease Control and Prevention (CDC) Heads Up website. In addition, parent respondents were likely to rely on youth sport coaches to inform them about their child's injury rather than their own knowledge about concussion signs and symptoms.

Parents want more information, meetings, and training programs for return-to-play procedures (Lyons et al., 2017). Bloodgood et al. (2013) found that concussion awareness is greatest among parents with children ages 10-13, suggesting that during this developmental stage when children are typically transitioning into high school sports, parents are seeking more information about concussions. As a result, increased awareness and education that is imparted to parents and caregivers before a child begins playing club and high school sports may be beneficial.

Research also suggests that income and education levels as well as advanced training may influence parental knowledge and attitudes toward concussions and return-to-play guidelines. Lin et al., (2015) found that concussion knowledge and attitudes regarding the management of concussions was significantly associated with high income and education levels. Of note, parents who experienced a diagnosed concussion themselves did not have higher knowledge and attitudinal scores than parents who did not experience a concussion. Gourley et al. (2010) found that parents who have had first aid training are more knowledgeable 
and better able to identify concussion symptoms. Return-to-play education and training is a way to reduce risks and facilitate post-concussion recovery in children who have experienced a concussion. Specifically, training in these areas could reduce the negative influences that hinder proper management and assist parents with seeking proper medical attention to identify and reduce health risks (Gourley et al., 2010; McGuckin, Law, McAuliffe, Rickwood, \& Bruner, 2016; Sullivan et al., 2009).

Concussion education and training for coaches, athletic trainers, and school personnel is a key component of concussion prevention and management. In addition, it is clear that parents and caregivers play a key role in concussion prevention and management. Yet, despite the growing awareness and knowledge of the signs/symptoms and seriousness of concussions, research demonstrates that there are still gaps in understanding the social and emotional symptoms related to concussions as well as the management of concussions (Kinnaman, Mannix, Comstock, \& Meehan, 2014; Mrazik, Bawani, \& Krol, 2011; Naftel, Yust, Nichols, King, \& Davis, 2014; Shenouda, Hendrickson, Davenport, Barber, \& Bell, 2012).

Most parents are aware that concussions are a serious injury to the brain yet are often unaware of return to learn (or return-to-school) and return to play protocols and state laws (Turner, et al., 2017). Moreover, while all 50 states have passed laws related to concussion and sport in school-age youth, most states have no law in place related to a return to school protocol (Thompson et al., 2016). A 2014 national poll conducted by researchers at the University of Michigan's C.S. Mott Children's Hospital found that roughly half of the parent survey respondents reported participating in some concussion education. Parents of children who play sports were more likely to receive concussion education versus parents of children who did not play sports. Further, the format of the concussion education made a difference in how confident parents were about making decisions regarding their child's concussion. The format parents rated as most useful was a video or live presentation; the least useful format was signing a waiver only (Clark, Kauffman, Singer, Gebremariam, \& Davis, 2014).

The issue of parent concussion knowledge and awareness is compounded by research demonstrating a reluctance on the part of high school athletes to report their concussion symptoms (Chrisman, Quitiquit, \& Rivara, 2013; Rivara et al., 2014). Parents often notice subtle changes in their child, however if they are well informed about concussion symptomology and risk, they can better advocate for their child. Therefore, the purpose of this study was to examine parent's perceptions and attitudes toward: 1) youth sport concussions; 2) the efficacy of returnto-learn and return-to-play educational resources; and, 3) a parent's role 
managing their child's return to school and play after a concussion.

\section{Methods}

This study utilized a grounded theory qualitative research design, which allows for the examination of perceptions and experiences of populations through the analysis of the data. Differing mechanisms and challenges of each participant required individual consideration of their experiences. In grounded theory, data analysis consists of reviewing the collected data, extracting repeated ideas, and coding those ideas. Researchers use constant comparisons of ideas with other instances (e.g. interview fragments) for similarities and differences. Thematic categories, which are groups of similar codes, become the basis for a new theory (Corbin \& Strauss, 2014).

Interviews conducted with parents examined their previous knowledge of concussions before their child sustained a sport-related concussion. The researchers also asked parents to compare their previous knowledge of concussions with their current perceptions and attitudes towards concussions. The interview questions assessed parental knowledge of the causes, symptoms, and postconcussion management of youth sport concussions. The researchers were also interested in any changes in attitudes or beliefs regarding the prevention and management of concussions. In addition, in order to determine whether the basis of each parent's concussion knowledge was from their child's experiences or their own, parents indicated if they had ever sustained a concussion themselves. Each interviewee shared unique individual experiences, allowing for deep examination and comparisons across families. The researchers' Institutional Review Board (IRB) granted approval prior to data collection.

\section{Participants}

This exploratory study utilized snowball sampling to recruit middle and high school participants for a study on the social and emotional symptoms of concussions that interfere with academic performance and relationships. Recruitment of these student participants occurred by gauging interest from people who knew of cases with potential abundant information (e.g. local educators, athletic trainers, or physicians). Researchers then used criterion sampling, to include all parent participants who met the following criteria: the participant's child sustained at least one concussion and experienced significant social/emotional symptoms.

From a sample of eight student participants, researchers interviewed six parents of children with histories of concussions; one parent's interview was not utilized since the cause of the child's concussion was non-sport related and the child was experiencing anxiety and emotional concerns prior to the concussion. Parent participants represented a diverse set of respondents. All five of the parent participants had 
youth sport participants who played in four different contact sports. Interviews of parent participants continued until researchers noticed similar repeated patterns with little additional information, indicating saturation of data (Corbin \& Strauss, 2014).

Parent demographics for the five participants included all females (four mothers, one stepmother) with children who sustained their concussion during an athletic event. The athletic events in which the concussions were sustained included lacrosse (2), basketball, soccer, and wrestling. Child demographics included both male and female student athletes ranging in age from 13-20 years (Table 1).

Table 1

\begin{tabular}{|l|l|}
\hline Parent Participant & Child's Concussion History \\
\hline $\begin{array}{l}\text { Ada (AM) is the mother of an 18-year- } \\
\text { old female. Ada has no history of a } \\
\text { diagnosed concussion. }\end{array}$ & $\begin{array}{l}\text { Ada's daughter sustained a concussion } \\
\text { playing soccer during her first year of } \\
\text { high school. Her daughter experienced } \\
\text { persistent concussion symptoms, includ- } \\
\text { ing headaches, memory difficulty, and sen- } \\
\text { sitivity to light and sound for three years. }\end{array}$ \\
\hline $\begin{array}{l}\text { Amber (AP) is the mother of a 17-year- } \\
\text { old female. Amber played soccer in her } \\
\text { youth and believes she experienced a } \\
\text { concussion, but never had a formal con- } \\
\text { cussion diagnosis. }\end{array}$ & $\begin{array}{l}\text { Amber's daughter sustained approximate- } \\
\text { ly three concussions while playing on her } \\
\text { high school lacrosse team, two of which } \\
\text { occurred three weeks apart. }\end{array}$ \\
\hline $\begin{array}{l}\text { Laura (LL) is the mother of a 16-year- } \\
\text { old female. Laura is a basketball coach } \\
\text { who previously received concussion } \\
\text { training. Laura has never had a diagnosed } \\
\text { concussion. }\end{array}$ & $\begin{array}{l}\text { Laura's daughter sustained two concus- } \\
\text { sions over the last year while playing for } \\
\text { her high school basketball team. }\end{array}$ \\
\hline $\begin{array}{l}\text { Mary (MP) is the mother of a 17-year- } \\
\text { old male. Mary has never had a diag- } \\
\text { nosed concussion. }\end{array}$ & $\begin{array}{l}\text { Mary's son sustained two concussions } \\
\text { over three years. The first concussion } \\
\text { occurred at the age of 13 after engaging } \\
\text { in an altercation with a football player. } \\
\text { His second concussion occurred at the } \\
\text { age of 14 during a statewide wrestling } \\
\text { match, after the opponent threw him to } \\
\text { the ground. }\end{array}$ \\
\hline $\begin{array}{l}\text { Renee (RM) is the mother of a 16-year- } \\
\text { old female. Renee has never had a di- } \\
\text { agnosed concussion but states that she } \\
\text { probably had one or more concussions } \\
\text { that went undiagnosed as a youth. }\end{array}$ & $\begin{array}{l}\text { Renee's daughter sustained a concussion } \\
\text { while playing goalie for her high school } \\
\text { lacrosse team. }\end{array}$ \\
\hline
\end{tabular}


Interviewees chose their interview session location to ensure convenience for participants. These locations included the researchers' conference room, the private section of a local restaurant, and the parents' homes. Each participant was assigned a pseudonym for confidentiality. In addition to their child's injury, parents discussed their own concussion history.

\section{Data Collection}

Data collected for this project were part of a larger study examining student concussions. The researchers collaboratively developed a semistructured interview guide with questions based on a review of relevant literature as well as the background experiences of each researcher. The first author has a research focus on youth sport, the second author has background experiences researching concussions in school-age children, and the third author has a research focus in anxiety. Trained research assistants conducted all parent interviews. The second author trained the two (2) research assistants to conduct these interviews. The training included practice interviews with each another, followed by practice interviews with two other parents who were not participants in the study. Recordings and revision of the practice interviews by the second author provided corrective feedback prior to data collection.

Parent interviews each lasted approximately 30 minutes. Digitally recorded interviews took place in a quiet location of the participants' choice. The interviews resulted in 86 transcribed pages of data. During the interviews, participants described their child's experiences with concussions and persistent symptoms; their concussion knowledge and attitudes towards prevention, recovery and management; factors or people that helped or hindered recovery; and their own concussion experiences. Each interview concluded with the interviewer summarizing key points and allowing participants to clarify or add information as needed. Interviews were transcribed word-forword; extracted fragments were later edited to remove unnecessary fillers (like, you know, um, just, I mean) to improve readability.

\section{Data Analysis}

The researchers analyzed interview data through an inductive analysis, searching the transcribed interviews for meaningful fragments. Fragments were useful for developing a categorization scheme and matching codes. The researchers then sorted the data using the codes to develop patterns and themes. Two reviewers conducted this step independently. After reviewing all of the parent interview transcripts independently, the two reviewers collaborated to identify overarching categorical themes. They then reviewed the data in order to sort it into thematic categories. The data was then subject to a re-working and re-coding process by all three researchers. In this way, the researchers, examined the participant 
responses and were able to generate new insights into the data and identify relationships among the categorical themes (Corbin \& Strauss, 2014).

Trustworthiness. Each interview concluded with a summary of the interview and a member check to determine if the summary was an accurate depiction of the participants' responses (Marshall \& Rossman, 2016). An iterative peer debriefing examination of the participant transcripts by multiple evaluators provided another means of confirming the reliability of the interview data. As noted previously, coding and re-coding of the transcripts ensured the dependability of the data analysis. Additionally, analysis of the data by a research team with various areas of expertise allowed for triangulation to enhance the credibility of the research (Krefting, 1991). As previously mentioned, the research team members have conducted research in the areas of youth sport, concussions in school-age children, and anxiety.

\section{Findings}

Analysis of the interview data resulted in five thematic categories regarding parental experiences with and perceptions of concussions before and after their child suffered a concussion. Themes that emerged from the interviews included levels of knowledge pre-concussion, changes in attitudes towards the prevention and management of concussions, varying symptoms, different management strategies, and the roles/relationships of various personnel involved throughout the incident and recovery process.

\section{Various Levels of Pre-concussion Knowledge}

Most participants knew little about concussions unless they previously had a directly linked experience or other outside training. As Amber commented, "I did not know a whole lot. I don't have a lot of experience with it."

Regardless of the prior experience with concussions, there were common ideas about concussions. Many expressed that experiencing more than one concussion is dangerous and after a certain number of concussive injuries, participation in sports or other types of physical activity is no longer an option. Amber claimed, "What I did hear was if you get one, you can play again. If you get two you're iffy. If you get three you're out." To elaborate, Mary said, "I knew nothing about concussions. I guess I knew you could get a concussion and I knew in sports in school after three, you're done. That was about it."

Similarly, there are common symptoms that people associate with concussions even without having any experience with a concussion. Amber pointed out:

I knew that you needed to look for their pupils to see if they were dilated unevenly, nausea, throwing up, that kind of thing and then you take them immediately to the doctor. 
Participants who had prior training and involvement in concussion education as a coach, sport official, or sport administrator articulated more detailed and accurate knowledge about them. For example, Laura said:

I am so knowledgeable because

I had taken the concussion [training] two or three times and I am a coach and a teacher. I knew that recovery time could vary and that you had to be symptoms free and they do that impact test.

Renee elaborated:

So I coached at [school name redacted], the freshman soccer team. You have to take the weird little complete five questions kind of thing. So I know that concussions are really dangerous when you have one to continue playing. If a kid gets hit in the head, take them out of the game. Make sure they're not dizzy, look into their eyes, right? When I coached and someone hit their head or fell they came out for the rest of the game.

\section{Changes in Attitudes}

Participants expressed that their own thinking about concussions changed after their child's injury and that other parents may not understand the impact and seriousness of concussions because of the variability and subtleness of some concussion symptoms. Mary remarked, 'Yeah. They don't realize it. People don't take it serious. Head injuries are the worst thing that you'd ever want your kid to deal with," and Ada explained, "I realize now what a huge impact it has on your life, the importance of educating schools on the impact that concussions have."

Many participants discussed how their style of parenting or their own perceptions regarding concussions influenced their attitudes toward their child after the child's concussion. Amber described a discussion she had with her husband that demonstrates discrepancies in attitudes toward concussions between parents:

Because my husband didn't go talk to the trainer or the doctor or anything like that he'd be like, 'Just shake it off, she's fine.' And I'm like, 'You know she's really not. If you pay more attention she's really not. It's going to take a little more time.' So he's like, 'You know you and I have had them,' And I'm like, 'Well maybe but, that was also twenty-five, thirty years ago.' Mary discussed how her changes in attitudes influenced her parenting and how she interacts with her child following the concussion. Specifically, she mentioned:

I'm one of those moms that's like, 'Get up, let's move, let's go. Move your butt, we're not going to sit around today.' I've had to redirect that to him. I can't be like, 'Get the hell up, get moving' because it breaks him down. I'll be like, 'Come on, you've got to get 
moving, let's go.' So I've had to change my parenting with him so he didn't take it so offensively. Additionally, Mary added:

I was always that mom that pushed. If you weren't first, you're last. I mean the year he dislocated his shoulder he wanted to play and I was like, 'Dude, if you can do ten pushups I'll let you go back out there.' [He] dropped down, he gave me ten... I was always pushing, pushing, pushing. I will say this: I wish I knew then what I know now.

Participants described how the personal experience of concussion recovery gives people a chance to form their own ideas. As Amber claimed:

I do think too that even if you haven't had a concussion, you probably have an opinion, but then once you experience the concussion, I think your opinion changes. Yeah, I think attitudes are changing.

\section{Variations of Symptoms}

Many participants noted various symptoms that their children had after sustaining a concussion. In addition to headaches and dizziness, all of the participants noted other physical symptoms that their child had after sustaining a concussion. Ada explained that her daughter, “... was bright red, her eyes were glassy, she was nauseous. She looked horrible." Amber described her daughter as "... really nauseous and out of it. She kept her head down between her legs, the lights were bugging her, she wobbled."

Participants also described various social/emotional symptoms that their child experienced after sustaining a concussion. These symptoms varied from anxiety, to depression and isolation, and irritability. Ada described her daughter as:

...exhibiting behaviors of OCD. Her room was immaculate, which was something that she had never done before. But I think her room became her bedroom so that's all she could control, what was in her room.

Ada also explained how her daughter was "... isolated more in her room back then than she ever did before because it was quiet," and Amber claimed her daughter ... 'felt very left out of things, she was pretty sad, and when she didn't bounce back quickly I think she got a little depressed and sad..."

Finally, other symptoms occurred including sleep issues. Two participants, Ada and Amber, described opposing symptoms concerning their daughters' sleep, respectively saying, "She didn't sleep well. So she never really felt rested," and, "She basically just slept the whole day." These two statements demonstrated the variability of symptoms across students. Memory issues can also arise because of a concussion. This was reinforced by Ada, who explained the difficulty her daughter faced: "She couldn't focus to read [and] she couldn't 
remember what she read." Similarly, Mary describes an incident between her son and his coworker, saying:

I guess I didn't realize the severity of it and [his coworker] was like, 'One day when we were working, he told me the exact same story twice and he didn't even remember that he had even told me it.'

\section{Different Management Strategies Continuation of play. One} important concussion management strategy related to sports is the continuation or discontinuation of play. Over half of the participants discussed their child immediately returning to play after the impact to their head. Mary describes the experience of her son immediately returning to a wrestling match after he was dropped on his head:

I looked up at him, his coach, and his coach gave me the thumbs up, so I gave him the thumbs up to go because we were just thinking [my son] was nervous. When he went back out on the mat, I noticed that he wasn't really going towards the ref, and the ref kind of had to lead him back. And he started wrestling again. He ended up losing... and he was done for the day. He got extremely upset and when he was getting his stuff I noticed he was falling around, but I thought he just had an attitude and he was mad.
Ada described the experience of her daughter immediately returning to play in her soccer game:

She continued to play. Her coach didn't take her out and one of her good friends kept saying, 'You need to take her out, she's stumbling.' So she called me when she got out of the game and she was laughing and almost manic-y. Saying 'Mom! I got a concussion! I'm totally fine! It's a double header; can I stay the whole time? I'm really, I'm fine!' ... So I could have gone to pick her up but I didn't because she sounded fine. I called my pediatrician who said 'As long as she's not profusely vomiting, don't worry about it.' Initially it seemed as if Ada's child was okay and able to return to her game. However, Ada proceeded to say: So then she calls me while I'm on the phone with the pediatrician. And at this point it's forty-five minutes later probably and she [said] 'Mom, I can't, I don't feel good. I can't keep my eyes open.' So I don't know why we did this but a friend of hers... drove her home. And she got to the house and she could barely walk. Her eyes were very glassy, she could barely stand.

Return to play. All participants discussed the process their child went through to return to school and playing their sport. All five participants 
mentioned that it was a long, gradual process. Amber describes her child's experience as follows:

The trainer at [her school] is really good about following a regimented return to play kind of thing. So she wouldn't let us return to play until we went back to the doctor and so I think she was off an entire week. And then I think I took her back either the Monday or Tuesday following, so it had probably been a week and a half, and they said she was cleared to just practice. So that consisted of fifteen minutes on a treadmill I think was the first day. The second day was an elliptical machine. And then the third day I think she ran around the track and she was able to throw the ball against the wall. And then the fourth day she could do drill with the team but she wasn't allowed to have any contact at all. And then the fifth day she was allowed to have some contact. And then after that I think she was allowed to return to play. So the rule was if she experienced any symptoms during any of those episodes, she would go back to zero and start all over.

Amber's experience demonstrates how school and healthcare professionals created a plan to ensure that her child was monitored and symptom free prior to returning to play. However, even if all the protocols for return to play are followed, there is still a chance that a child may sustain a second concussion. Laura described such a situation after her daughter experienced multiple concussions while playing high school basketball:

She kept trying to come back and doing the steps to re-enter into play and she would do a little bit, do a little, bit, then she would have the symptoms again. So she ended up and they took her back to the main concussion people at [name of hospital redacted]. And he basically said that she was having post-concussion-like syndrome... [She] had an appointment when she got back from the cruise. And they were kinda week-by-week touch basis on how she was doing. So it took two more weeks to kinda get her cleared... until there were no more symptoms at all for a certain amount of time. She was probably good for about three games or so and you know, now we are totally out of shape and all this other kind of good stuff, but then she got hit in the nose again and face again with that other girl. So that's when we just pulled the plug and said that was it.

\section{Roles and Relationships}

Athletic staff. Often the first responders after a child has sustained a concussion while playing a sport are the athletic staff, specifically athletic trainers and coaches. High school athletic trainers and coaches in all 50 states are now 
required to receive training in recognizing the signs and symptoms of concussions. Many participants discussed how the athletic trainer and/or coach were the most helpful during their child's recovery from concussion. Amber, talking about her daughter's recovery, claimed:

I think the most helpful was the trainer because she was extremely knowledgeable. She wasn't really concerned about getting her back into play more so than she really wanted her to be healthy.

Laura said of her daughter's recovery and return to basketball, "Well her trainer was very involved with things and monitoring how she was doing." However, there are incidents in which the athletic staff do not follow concussion protocol and instead allow the child to continue playing in the game or match. Mary described her experience with a wrestling coach who wanted her son to continue wrestling after sustaining multiple concussions due to his abilities and previous success:

I was kind of upset because after the ref stopped the match the one coach on the sideline waved him on and said to go ahead, that it was his contacts. My son has never worn contacts and they know that. And they know the severity of his concussions that he's had. And so he kept going, so I had to physically go down on the mat and stop the match. Well then the trainer came over and she looked at him and I had already told the coaches that I was taking him regardless of what they had said.

School staff. Along with the athletic staff, the entire school staff has an important role to play in ensuring a smooth transition back into the classroom and the overall school environment. Participants described challenges when school staff was unresponsive or failed to communicate with each other For example, Mary stated:

Last year, well up until then it has been a fight. The communication throughout the school has not been there. I went in, I talked to the principal, I talked to their teachers. We've had some issues... So we have had some issues and probably three, four months into school I finally decided I'd had enough and I called a meeting and I had all his teachers be able to be present and I kind of broke down what he was going through. And the teachers were like, Oh well we didn't know it was like that and we weren't being aware of it and I kind of called the principal out and I was like I've been sending emails and calling you and I'm telling you this stuff... Amber described the experience of battling with the school's administration to get permission for her daughter to wear a hat and sunglasses while in school:

And so as far as wearing the glasses and that kind of stuff 
[the administration] said, 'Well we need a doctor's excuse for that.' And I called them and said, 'No. We went and she was confirmed having a concussion. The trainer took her out. She has a concussion. You need to let her do what she needs to do. And when I go to the doctor I'll give you the information but for right now this is what's happening.' And they said, 'Fine.' But they wanted me to get paperwork to back it up.

Often there are people within the school willing to help and accommodate. Amber, the same parent who had to argue with the school administration about accommodations, discussed how the assistant principal was accommodating and helpful by allowing her daughter to make-up several missed tests:

There was an assistant principal that was in charge of like the testing and that kind of thing. And he was really accommodating. He was actually at both of the games when it happened. And saw what happened and so, the first time the trainer went right to him and said, 'She will not be there tomorrow to take these tests.' And then the second time he was there and he was actually sitting by me. So I went and introduced myself and was like, 'Um, just FYI we won't be at school tomorrow.' So he was like, 'No problem. There's another date, we'll just make it happen that date.'

\section{Medical professionals/specialists.} Several parents expressed frustration with the discharge instructions and medical care that was provided to their child after sustaining a concussion. Parents were sometimes unsure of what type of physician or healthcare provider to take their child to and were sometimes given conflicting information and instructions from different medical professionals. For example, Ada described her experience dealing with the emergency room doctor:

We got into the room and within two or three minutes of getting there, a nurse comes in and said, 'The doctor diagnosed you with a concussion and gave you medicine.' Now, we never saw the doctor at that point. But they said, 'Just walking down to the room, they knew she had a concussion.' So they gave her something. I don't know if it's a antinausea thing. I don't really remember what they gave her but they gave her something. And I just remember thinking, 'That's really bizarre because they didn't even come in and see us.'

Ada later on in the interview claimed:

Nobody really knows what they're talking about. I mean that's it. We went to [name of hospital redacted] [and] they told us one thing. We went to [name of 
hospital redacted] in [another city], they told us something the exact opposite. People do not understand the brain.

Since concussions are an "invisible" injury with multiple symptoms - cognitive, physical, social, and, emotional - parents received conflicting instructions regarding proper concussion recovery from different doctors. The involvement of multiple specialists in a treatment plan may be necessary for optimal concussion recovery, but it can also lead to confusion for parents. Renee explained how her daughter was seen by several different doctors and specialists:

So we went to Dr. $\mathrm{M}$ and he referred us to Dr. A at [name of hospital redacted]. My brotherin-law is the medical director for the [name of professional baseball team redacted]. We were talking to him and he told us that we should see Dr. C...So we have also seen a neuropsychologist...she saw a vestibular doctor .... and then also PT...She's currently seeing a vision therapist... [and] she had blood tests with the neurologist.

Parents. As noted throughout this section, parents often find themselves in conflicting positions when it comes to interacting with different school and medical personnel, but parents also struggle when it comes to their own feelings and ideas of what to do with their child. Mary described her feelings after her son's concussion and the importance of recognizing the best interests of the child:

I think sometimes as parents when you have a kid that's an extraordinary athlete and you know that they have something and we push a little bit harder, maybe try not to push so much. Really pay attention to your kid. If you're noticing that they're sleeping, you notice that they're fatigued, you're noticing that their moods are changing, those are symptoms that people ignore all the time 'cause we dismiss them as hormonal or they're just teenagers. Like I said, if I would have known then what I know now, [my son] would have never touched a football.

Parents often struggle due to the lack of available or adequate education. They receive some concussion information but they receive little to no information about what they should do to advocate for their child if he or she sustains a concussion. Mary validated this, exclaiming:

...The smack of reality is I truly believe there's not enough education. We have to as parents read a paper and we have to watch a movie... [but] it doesn't give you an example and I'm hoping to someday go in and talk to the parents and tell them. 


\section{Discussion}

Interviews with parents of children who sustained a concussion or multiple concussions revealed gaps in the parents' knowledge of how to recognize and manage concussions. This finding is consistent with the results of a review of research articles published from 2009-2016 on concussion knowledge among youth sport stakeholders which found knowledge gaps in parental understanding of proper medical care for concussions and return-to-play protocols (Kim, Connaughton, Leeman \& Lee, 2018). The results of the current study demonstrate that this knowledge gap persists despite an increase in education and training programs for youth sport participants, coaches, officials, and parents. Of particular note are the concerted training and marketing efforts by the CDC, through its Heads Up public health campaign, to educate and train parents, coaches, school professionals and health care providers on the risks of concussions (CDC, 2017).

This finding is also consistent with the results of several recent studies that examined parents' concussion history (Kay, Register-Mihalik, Ford, Williams, \& Valovich McLeod, 2017) and parents' health literacy (Turner, et al., 2017) as determinants of parental knowledge of concussions and return-to-play protocols. Both studies found that despite increased awareness and education related to sport concussions, parents still have misconceptions about proper returnto-learn and return-to-play procedures.
It is critical to acknowledge that these gaps in parental knowledge exist in order to provide appropriate concussion education for parents.

Based on the results of this study parents seem to need more information and knowledge about both the physical and social/emotional symptoms of concussions. Parents often relied on coaches to inform them that their student athlete suffered a concussion as well as how to manage their return-toplay. Yet, they also perceived that athletic staff, school staff, and even medical professionals were sometimes confused as to the proper treatment, management of concussions, and return to- learn and play protocols. Improved training for athletic (i.e. coaches, athletic trainers) and school personnel is important as well as better coordination of postconcussion care and return to-learn and play guidelines. Further, parents need to know how to advocate for their young student athletes as knowledge about the prevention, treatment, and management of concussions increases and changes over time.

Parental misunderstandings regarding the chronic symptoms of a concussion and inconsistencies in the management of concussions may also be the result of inadequate or ineffective education efforts. Parents in our study were aware of the acute physical signs and symptoms of a concussion but often not aware of how to manage the lingering physical and emotional/social symptoms of a concussion. There has been a lack 
of research on the effectiveness of sport concussion education for parents and young athletes (Turner, et al., 2017).

Further, parents in our study reported a change in their attitudes toward concussions and the management of concussions only after their young athlete had sustained a concussion. This finding suggests that current educational efforts regarding concussions may not be effective in changing parental attitudes and behavior related to the prevention and proper care of concussions (Kay, et al., 2017). More research on the effectiveness of sport concussion education, appropriate formats for delivering this education to parents, and how knowledge about concussions can translate into attitudinal and behavioral changes are important next steps in the prevention, proper care and management of youth sport concussions.

\section{Recommendations}

\section{Increase knowledge about} definition and symptoms. According to research, most people can identify the physical signs and symptoms of a concussion (Coghlin et al., 2009; Gourley et al., 2010). It is clear from the results of this and other studies that there are several different symptoms of a concussion, with many participants' children experiencing similar symptoms such as headaches and nausea, but also social and emotional symptoms (i.e., anxiety and depression). While some effects of a concussion are immediate and noticeable, as demonstrated by the first participant (Ada), it is extremely important for parents and other caregivers to know that some symptoms are more subtle and not easily identified. While some children may initially seem fine, they can still suffer emotional and social symptoms not manifested immediately after the incident. Not everyone experiences the same symptoms, so it is important to provide information concerning all potential symptoms and their effects. This, in turn, leads to better diagnoses and care plans, as well as mitigating the risks associated with sustaining multiple concussions (Davis et al., 2017).

\section{Improve training for athletic and} school staff. While more education is important for parents, consistent training for school and athletic staff would be beneficial. Participants discussed the inconsistencies in the decisions and recommendations made by athletic trainers, coaches, and school administrators in following return to learn and return to play protocols. While athletic trainers gave instructions for concussion prevention and recovery, parents in this study reported that coaches sometimes pressured athletes to return to play before they fully recovered. Similarly, parents described incidents where school personnel did not provide appropriate accommodations for their child. It is essential that athletic staff and school personnel are following appropriate concussion management practices and communicating with each other to ensure adequate recovery. 
Proper training as well as a team-based approach would result in more consistent practices and improved communication among athletic staff and school personnel.

Coordination of care. As

demonstrated in the data collected in this study, athletic staff, school personnel, and healthcare professionals have different depth and breadth of experience with concussions. This may create confusion for parents who are trying to help their child. In order to best help the child, it is important to have parents, athletic staff, school staff, and healthcare professionals in communication with each other, working together to create a coordinated plan for the child to return to learn and return to play.

\section{Parents as advocates for their} children. As much as parents sometimes rely on their child's coach, athletic trainer, or even the child themselves to make return to play decisions, parents and caregivers must actively participate in these decisions. Previous studies have shown that high school athletes will say they are feeling fine or not report concussion symptoms in order to stay in a game (Chrisman, Quitiquit, \& Rivara, 2013; Rivara et al., 2014). This study revealed that some parents struggled at first to advocate for their child, however, most agreed that it is important to advocate for a child who has suffered a concussion and how they wish they had done so sooner.

\section{Advocacy and support groups.} Inconsistencies in concussion management may be the result of poor communication and/or support among people who have gone through the experience. This is especially true for parents of children with concussions. Research has shown that parents want more information and opportunities to meet and discuss concussions and management practices (Clark et al., 2014; Lyons et al., 2017), and this was reiterated among participants in the current study. One way for this to happen would be to encourage parents to join advocacy groups such as Mothers Against Concussions or to create their own support groups to describe their experiences and serve as advocates for other parents who find themselves in similar situations.

\section{Limitations}

The low number of participants allowed for an in-depth examination of their experiences, but limits generalizability. All participants were from one Midwestern state, limiting the generalizability across different regions of the United States. Additionally, the number of concussions each participant's child experienced and the amount of time that occurred between the concussion and the interview varied, leading to inconsistencies in responses. Another limitation of the study was the potential for bias. Due to the nature of the questions and the face-to-face interviews, the parent participants may 
have answered the researcher's questions in socially acceptable ways.

\section{Conclusion}

The purpose of this study was to gain a deeper understanding of the concussion experiences, attitudes, and perceptions of parents of children who have sustained a concussion. We also examined parents' perceptions of the effectiveness of return-to-learn and return-to-play educational resources as well as how they understood their role managing their child's concussion. Future studies could examine the behavioral changes needed to more effectively prevent and manage concussions. This could include changes in parent attitudes regarding sport participation and concussions.

Future research might examine the level of knowledge and attitudes towards concussions in other school and medical personnel. Parent participants most often cited the involvement of coaches, athletic trainers, and medical specialists in the management of their child's concussion. However, there remain inconsistencies in the identification and management of concussions from these various school and medical personnel.

\section{Acknowledgement}

The authors would like to thank Rachael Rockwell for her help with data collection and this manuscript. Funding for this project came from the Ohio Department of Public Health, Division of Emergency Medical Services.

\section{References}

Bloodgood, B., Inokuchi, D., Shawver, W., Olson, K., Hoffman, R., Cohen, E., Muthuswamy, K., M.P.H. (2013). Exploration of awareness, knowledge, and perceptions of traumatic brain injury among American youth athletes and their parents. Journal of Adolescent Health, 53(1), 34-39. DOI: https://doi.org/10.1016/j. jadohealth.2013.01.022

Centers for Disease Control. (2017). HEADS UP to brain injury awareness. Retrieved from: https:/ / www.cdc.gov/HEADSUP/

Chrisman, S.P., Quitiquit, C., \& Rivara, F.P. (2013). Qualitative study of barriers to concussive symptom reporting in high school athletics. Journal of Adolescent Health, 52(3), 330-335. DOI: 10.1016/j. jadohealth.2012.10.271

Clark, S.J., Kauffman, A.D., Singer, D.C., Gebremariam, A., \& Davis, M.M. (2014). Head smart? Parents get confidence boost from concussion education. C.S. Mott Children's Hospital National Poll on Children's Health, University of Michigan, 22(2). Coghlin, C. J., Myles, B. D., \& Howitt, S. D. (2009). The ability of parents to accurately report concussion occurrence in their bantam-aged minor hockey league children. Journal Of The Canadian Chiropractic Association, 53(4), 233-250. 
Corbin, J. M., \& Strauss, A. L. (2014). Basics of qualitative research: Techniques and procedures for developing grounded theory ( $4^{\text {th }}$ ed.). Los Angeles: Sage.

Davis, G., Anderson, V., Babl, F., Gioia, G., Giza, C., Meehan, W.,... Zemek, R. (2017). What is the difference in concussion management in children as compared with adults? A systematic review. British Journal of Sports Medicine, 51, 949-957.

DOI: http://dx.doi.org/10.1136/ bjsports-2016-097415

Gourley, M. M., Valovich McLeod, T. C., \& Bay, R. C. (2010). Awareness and recognition of concussion by youth athletes and their parents. Athletic Training \& Sports Health Care, 2(5), 208-218. DOI: https://doi. org/10.3928/19425864-20100524-03

Institute of Medicine and National Research Council. (2014). Sportsrelated concussions in youth: Improving the science, changing the culture. Washington, DC: The National Academies Press. DOI: https://doi.org/10.17226/18377

Kay, M.C., Register-Mihalik, J.K., Ford, C.B., Williams, R.M., \& Valovich McLeod, T.C. (2017). Parents' and child's concussion history as predictors of parental attitudes and knowledge of concussion recognition and response. The Orthopedic Journal of Sports Medicine, 5(12), 1-11. DOI: 10.1177/2325967117742370

Kim, S., Connaughton, D.P., Leeman, R.F., \& Lee, J.H. (2018). Concussion knowledge of youth sport athletes, coaches, and parents: A review. Journal of Amateur Sport, 4(1), 82-107. DOI: https://doi.org/10.17161/jas. v4i1.7579

Kinnaman, K. A., Mannix, R. C., Comstock, R. D., \& Meehan, W. P. (2014). Management of pediatric patients with concussion by emergency medicine physicians. Pediatric Emergency Care, 30(7), 458-461. DOI: 10.1097/ PEC.0000000000000161

Krefting, L. (1991). Rigor in qualitative research: The assessment of trustworthiness. American Journal of Occupational Therapy, 45(3), 214-222. DOI:10.5014/ajot.45.3.214

Lin, A. C., Salzman, G. A., Bachman, S. L., Burke, R. V., Zaslow, T., Piasek, C.Z., Upperman, J. S. (2015). Assessment of parental knowledge and attitudes toward pediatric sports-related concussions. Sports Health: A Multidisciplinary Approach, 7(2), 124-129. DOI: 10.1177/1941738115571570 Lyons, V. H., Moore, M., Guiney, R., Ayyagari, R. C., Thompson, L., Rivara, F. P., Vavilala, M.S. (2017). Strategies to address unmet needs and facilitate return to learn guideline adoption following concussion. Journal of School Health, 87(6), 416426. DOI: https://doi.org/10.1111/ josh.12510

Macdonald, I., \& Hauber, R. (2016). Educating parents on sports-related concussions. Journal of Neuroscience 
Nursing, 48(6), 297-302. DOI:

10.1097/JNN.0000000000000212

Marar, M., McIlvain N., Fields S., \&

Comstock R.D. (2012). Epidemiology

of Concussions Among United States

High School Athletes in 20 Sports.

American Journal of Sports Medicine,

40(4):747-755. DOI: https://doi.

org/10.1177/0363546511435626

Marshall, C., \& Rossman, G. (2016)

Designing qualitative research (6th ed.). Thousand Oaks: SAGE.

McGuckin, M. E., Law, B., McAuliffe, J., Rickwood, G., \& Bruner, M. W. (2016). Social influences on return to play following concussion in female competitive youth ice hockey players. Journal of Sport Behavior, 39(4), 426445.

Mrazik, M., Bawani, F., \& Krol, A.L. (2011). Sport-related concussions: Knowledge translation among minor hockey coaches. Clinical Journal of Sport Medicine, 21(4), 315-319. DOI: 10.1097/JSM.0b013e31821e2b78

Naftel, K.G., Yust, E.M., Nichols, M.H., King, W.D., \& Davis D. (2014). Knowledge and management of sports concussions among coaches and certified athletic trainers in Alabama. Southern Medical Journal, 107(7), 418-423.DOI: 10.14423/ SMJ.0000000000000136

Rivara, F. P., Schiff, M. A., Chrisman, S. P., Chung, S. K., Ellenbogen, R. G., $\&$ Herring, S. A. (2014). The effect of coach education on reporting of concussions among high school athletes after passage of a concussion law. American Journal of Sports Medicine, 42(5), 1197-1203. DOI: https://doi. org/10.1177/0363546514521774

Shenouda, C., Hendrickson, P., Davenport, K., Barber, J., \& Bell, K. R. (2012). The effects of concussion legislation one year later - what have we learned: A descriptive pilot survey of youth soccer player associates. PM\&R, 4(6), 427-435. DOI: https:// doi.org/10.1016/j.pmrj.2012.02.016

Sullivan, S.J., Bourne, L., Choie, S., Eastwood, B., Isbister, S., McCrory, P., Gray, \& A. (2009). Understanding of sport concussion by the parents of young rugby players: a pilot study. Clinical Journal of Sport Medicine, 19(3), 228-230. DOI: 10.1097/JSM. Ob013e3181a41e43

Thompson, L. L., Lyons, V. H., McCart, M., Herring, S. A., Rivara, F. P., \& Vavilala M. S. (2016). Variations in state laws governing school reintegration following concussion. Pediatrics, 138, 1-7. Retrieved from www.aappublications.org/news Turner, R.W., Lucas, J.W., Margolis, L.H., \& Corwell, B.N. (2017). A preliminary Study of youth sport concussions: Parents' health literacy and knowledge of return-to-play protocol criteria. Brain Injury, 31(8), 1124-1130. DOI: 10.1080/02699052.2017.1298003 